\title{
Startup of an Industrial Adiabatic Tubular Reactor
}

\author{
J. W. Verwijs and $H$. van den Berg \\ Process Development \& Control Dept., Dow Benelux N.V., 4530 AA Terneuzen, The Netherlands \\ K. R. Westerterp \\ Chemical Reaction Engineering Laboratories, Dept. of Chemical Engineering, Twente University of Technology, \\ 7500 AE Enschede, The Netherlands
}

\begin{abstract}
The dynamic behavior of an adiabatic tubular plant reactor during the startup is demonstrated, together with the impact of a feed-pump failure of one of the reactants. A dynamic model of the reactor system is presented, and the system response is calculated as a function of experimentally-determined, time-dependent, manipulated variables. The values of model parameters are estimated by using the SimuSolv (1991) computer program. The data set collected during the reactor start-up is used for the parameter estimation procedure. An excellent agreement is obtained between the experimental and the calculated system response. Many continuously-operated commercial reactors require a complete conversion of one of the main reactants at the reactor exit. It is shown that for an industrial tubular reactor a much higher initial reactor temperature is required during the startup, compared to the reactor inlet temperature during normal steady-state operation, to ensure a complete reactant conversion. Much more research is necessary to determine whether this is a generally valid rule.
\end{abstract}

\section{Introduction}

Higher safety standards, more stringent environmental regulations, better management of energy and raw materials, and increased product quality requirements have caused the structure of continuously-operated chemical processes to become increasingly complex and the process operating limits tightened. Therefore, understanding of the physical and chemical phenomena is required to set up safe, simple and effective operating procedures, both for steady-state and for dynamic operations. Process control is critical to running a continuouslyoperated chemical plant successfully within the operating limits, but basically the process control system is a translation of the strategy of "how to run the process" into controllers and related subjects. Therefore, operating procedures (specification of the equipment operation) are key issues for a successful operation, because they are intermediates between process design (equipment specification) and process control (equipment operation).

Correspondence concerning this article should be addressed to $\mathrm{K}$. R. Westerterp.
Operating procedures for continuously-operated chemical plants consist of two parts: the first part describes the procedures during normal operation (steady-state operation); the second describes the procedures for start-up, switch-overs, and shutdown (dynamic operation). Often operating limits during the normal operation are determined primarily by the process economics. Hence, during the past decades, the chemical industry has put much effort on steady-state process optimization, resulting in increased process yields, improved product quality, and a reduced energy consumption and environmental pollution.

The most complex problems in defining operating procedures is to quantify the process dynamics and to specify the operating limits during dynamic operation. Usually in a process, a number of variables are available which can be adjusted freely within the operating limits by the plant operator and/ or the process control system. The selection of correct variables for control purposes, along with the specification of the operating windows of these variables, can be a crucial step. Also 
the sequence of putting equipment into or out of service, together with the necessary optimum process-related conditions, and the impact of unexpected process interruptions due to equipment failures bear the same level of importance.

Most published studies on tubular reactor dynamics are theoretical and concerned with the characterization of the phenomenon taking place in fixed-bed reactors. Some studies available take operating limits into account.

Hahn et al. (1971) studied the start-up of a jacketed tubular reactor from uniform initial conditions to predefined steadystate conditions by manipulating the wall temperature of the reactor. A distributed maximum principle is used to drive the system from the initial state toward the final steady state by minimizing the spatial integral of the weighted sum of the squared concentration and temperature deviations from the desired steady-state profiles, integrated over the transient startup period of a fixed length. The proposed method can also be used when operating limits have to be taken into account.

Mann et al. $(1979,1980)$ developea a dynamic model of a $\mathrm{SO}_{2}$ fixed-bed oxidation reactor to determine improved startup procedures, which are required to reduce $\mathrm{SO}_{2}$ emissions to atmosphere during the start-up of a sulphuric acid plant. A number of model simulations were performed to determine the influence of some variables, and significant differences are found on $\mathrm{SO}_{2}$ emission levels. The suggestion is made to determine the optimal mode for the manipulated variables during start-up via the calculus of variations and Pontryagins maximum principle. Laboratory studies were carried out by Mann et al. (1986) to prove some model assumptions. It did not lead to principle changes in the philosophy to define start-up procedures compared to the preceeding studies of Mann et al. $(1979,1980)$.

In general, the reactor dynamics including reactor start-up are studied within a certain mathematical framework, such as impact of different types of boundary conditions and step changes of adjustable variables. Often laboratory experiments are carried out to prove the theoretical assumptions for heatand mass-transfer mechanisms in the catalyst bed and/or particles, or to prove the predicted system response as a function of the variables studied within the boundaries of the indicated mathematical framework, without a direct link to industrial practice. This "limited research scope" has to be extended to operating procedure synthesis and nonsteady-state process control to reduce environmental pollution and to improve process safety, because most accidents in chemical plants occur during dynamic operation (Amundson et al., 1988). Haastrup (1983) concluded from a study of accident case stories that the frequency rate, on a time basis, of design related accidents in continuously-operated chemical plants seems to be at least one order of magnitude higher during dynamic operation than during normal running conditions. Therefore, the current process engineering practice of designing continuously-operated chemical plants for steady-state running conditions poses a related research problem for operating procedure synthesis and nonsteady-state process control (Amundson et al., 1988).

Two types of knowledge can be used in plant-wide operations (Stephanopoulos, 1988):

a) Declarative knowledge, based on first principles, characterizing the behavior of processing units

b) Procedural knowledge, representing the methodologies employed by plant operating personnel and process control systems to operate the process.

Both types of knowledge are required to study operational aspects of a reactor startup. A dynamic process model, based on first principles, should answer questions about reactor behavior and operating limits. A representation of the operating procedures should answer questions about "how" and "when" to start up a reactor system, because a reactor startup cannot be isolated from an entire plant startup.

To our best knowledge, no results are published about reactor dynamics and operating procedures of commercial reactor systems. This article describes the startup of an industrial adiabatic reactor, as well as the impact of a failure of the feed pump of one of the reactants. A dynamic model, characterizing the behavior of the system, is presented, and the values of the model parameters are estimated by using the SimuSolv (1991) computer program. Some research results on the synthesis of reactor operating procedures for the startup of a continuouslyoperated chemical plant will be discussed in a future article.

\section{Plant Reactor System}

The plant reactor system consists of a feed mixer, a preheater and a series of seven horizontal vessels with baffles. The first vessel is bigger in size than the others, which have all the same dimensions. A sketch of the reactor is given in Figure 1. Also indicated are the locations of the relevant flow devices $(F)$ and thermoelements $(T)$. The reactor is insulated and located outdoors in an open structure. The total volume of the mixer and preheater is less than $1 \%$ of the total reactor volume. Basically the reaction starts already in the mixer, but the reactant con-

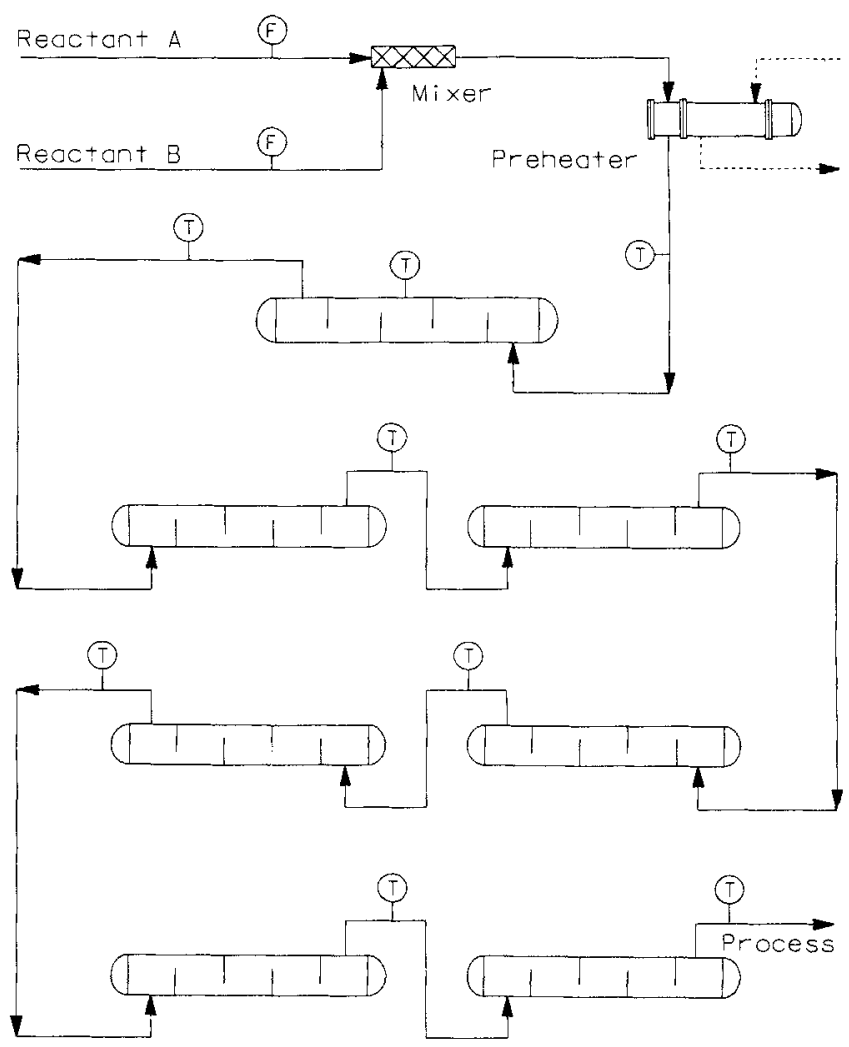

Figure 1. Plant reactor. 
version in the mixer and preheater will be very small due to the relatively short residence time and low process temperatures. Hence, the thermoelement in the preheater outlet is considered as being the location of the reactor inlet.

Thermoelements are located at several positions between reactor inlet $(z=0)$ and reactor outlet $(z=1)$. The relative position $(z)$ of a thermoelement is calculated by taking the ratio of the volume from the reactor inlet up to the particular thermoelement and the total reactor volume, according to the model assumptions.

The operation of the reactor system is computer-controlled, including the startup or shutdown of pumps and opening or closing of block valves (not indicated in Figure 1). Process data can be logged at regular time intervals from the process control computer.

For this study, the whole scheme of reactions carried out in the plant reactor is simplified to one overall reaction that describes the consumption of the main reactant $B$ :

$$
A+B \rightarrow C
$$

This exothermal reaction is carried out in the liquid phase at a pressure level sufficiently high to avoid boiling. Reactant $\boldsymbol{A}$ is fed in excess, because reactant $B$ should be totally converted at the reactor exit. The excess amount of reactant $A$ is also used to absorb the heat of reaction and limit the adiabatic temperature rise.

The conversion level of reactant $B$ is the most important operating requirement. Therefore, a minimum average reaction rate and a sufficient residence time in the system are necessary to ensure the required conversion level of reactant $B$ during reactor operation. Other operating limits resulting from the process economics are beyond the scope of this article.

\section{Reactor Startup}

The reactor system is filled up with a mixture of reactant $A$ and final product $C$ from storage before startup to create a mixture in the reactor that can be processed by the downstream plant section. This is done via the reactor feed system and with the preheater in service. During this period, the inert gases present in the reactor system are vented to atmosphere via the downstream plant section. This step is necessary only when the system had been emptied for inspection and/or maintenance, and can be skipped from the startup sequence if the reactor was not emptied beforehand.

First, reactant $A$ is fed into the reactor. Reactant $B$ is added into the system, and the preheater is put into service at the same moment to control the reactor inlet temperature, when the flow of reactant $A$ has reached the required setpoint.

The flow of reactant $A$ is controlled in ratio with the flow of reactant $B$, as soon as the flow of reactant $B$ has reached a certain minimum flow setting. The total reactor feed is increased to minimum plant capacity, if the flow of both reactants is at the required initial setpoints and the outlet temperature of the reactor is above a certain minimum temperature limit.

The flow of both reactants as a function of the dimensionless time $\sigma$ is shown in Figure 2. The dimensionless variables are defined in the Notation Section. In this figure the flow is expressed as a percentage of the particular flowmeter range.

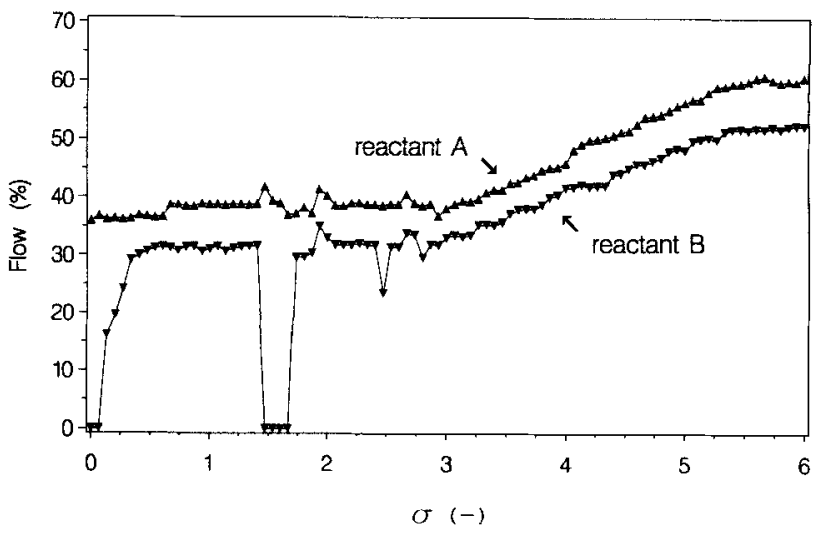

Figure 2. Observed flow of reactant $A$ and $B$ as a function of time.

The time $\sigma$ is scaled by taking the time origin $(\sigma=0)$ just before reactant $B$ is fed into the system. The flow of reactant $A$ at $\sigma=0$ is already at the required capacity. At $\sigma=0.10$, the feed pump of reactant $B$ is started up. During the period $\sigma=1.47$ until $\sigma=1.70$, no reactant $B$ is fed into the system due to a pump failure. The reactant $B$ feed pump is restarted at $\sigma=1.70$. At $\sigma=3.00$, the reactor feed is ramped up to a minimum plant capacity which is reached at $\sigma=5.50$.

The initial dimensionless temperature profile $\theta(z, 0)$ at $\sigma=0$ over the entire reactor is shown in Figure 3. The dots in this figure are the actual data, and the solid line is an approximation of the temperature values between the thermoelements, according to the Akima (1970) method to produce a smooth curve. These approximated values are used in the model of the reactor system.

The initial temperature profile depends on the following important conditions:

- The way the system is preheated and filled up by the plant operator

- The period between filling or reactor stop and the actual reactor startup in view of the heat loss to the environment

- The time span between the start of the reactant $A$ feed pump and putting the reactant $B$ feed pump into service

- The temperature and amount of reactant $A$ fed into the system before starting the reactant $B$ feed pump.

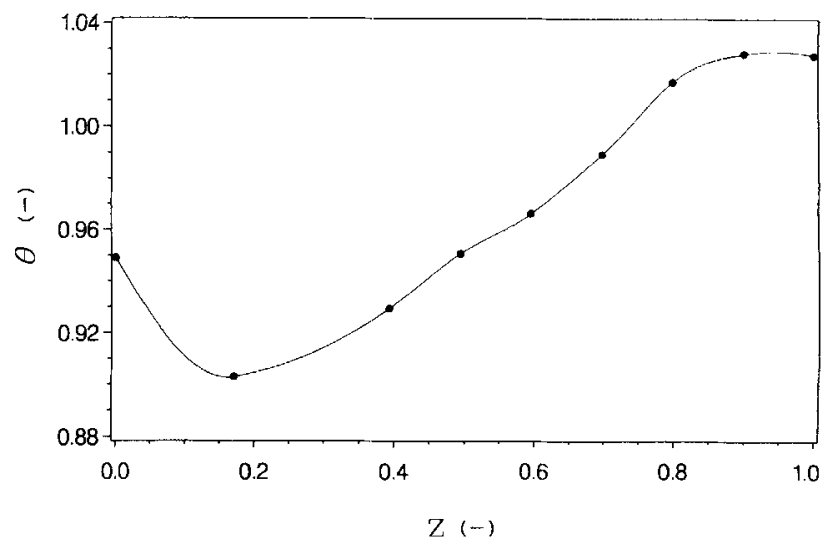

Figure 3. Temperature profile over the entire reactor length at $\sigma=0$. 


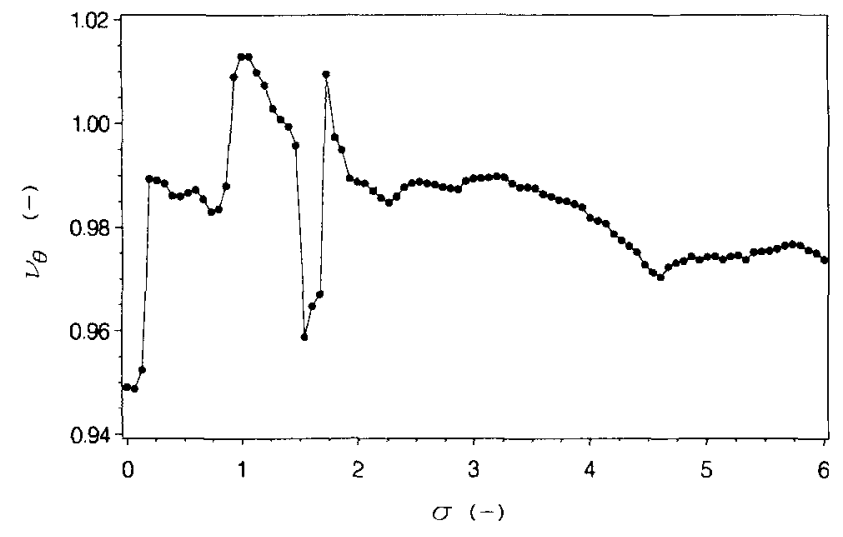

Figure 4. Observed reactor inlet temperature as a function of time.

The dimensionless reactor inlet temperature $\nu_{\theta}$ as a function of time is shown in Figure 4. The shape of this curve is influenced strongly by the step changes in the total flow through the system due to the start or stop of the reactant $B$ feed-pump and putting the reactor preheater into or out of service at the same moment.

The temperature $\theta$ over the entire reactor is shown in Figure 5. In this figure, the lines parallel to the $\sigma$-axis represent the response of the thermoelements at the dimensionless location $z$, and the lines parallel to the $z$-axis connect the data at the same moment. The overall error of the data is estimated to be $\leq 5 \%$ for the flow of reactant $A, \leq 3 \%$ for the flow of reactant $B$, and $\leq 0.5 \%$ for the temperature.

\section{Reactor Model}

The performance of this plant reactor indicates that a tubular reactor model can be used to describe the system. The following assumptions are made to define the model:

1. The reactor volume used in the model is equal to the total volume of the vessels and the piping in the system.

2. To represent the plant reactor as an empty tube reactor, the inside tube diameter $\left(d_{1}\right)$ or tube length $(L)$ should be

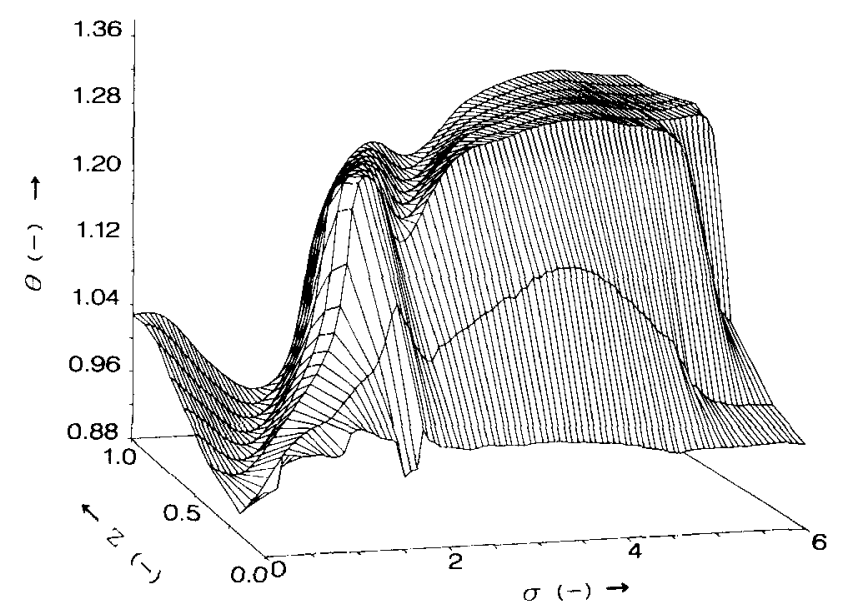

Figure 5. Observed reactor temperature vs. reactor lo. cation and time. defined. The tube diameters of six equal-sized vessels are used to calculate the tube length.

3. Approximately $8 \%$ of the heat produced by reaction is absorbed by the vessel wall during startup. Hence, the energy take-up in the reactor vessels is included in the model. The total amount of construction materials of the vessels and the piping is used to calculate an average outside tube diameter $\left(d_{2}\right)$ to be used in the model. The heat transfer coefficient $(U)$ between fluid and tube wall is assumed to be constant. Heat transported through the tube wall by conduction in the axial direction is neglected.

4. Heat take-up in the insulation blanket and heat losses to the surroundings are neglected.

5. Due to the system geometry the actual flow pattern will not be plug flow. The deviation of plug flow will be described by axial dispersion. There is no reason to distinguish between axial and radial dispersion in the model, due to the simplification of the system geometry. Mass $\left(D_{a x}\right)$ and heat $\left(\lambda_{a x}\right)$ dispersion coefficients are assumed to be constant over the entire reactor length despite the geometry variations.

6 . The reaction scheme is simplified to one reaction, describing the consumption of reactant $B$. This reaction is assumed to be irreversible and first-order with an Arrheniustype rate constant $\left(k_{1}=k_{10} \cdot \exp \left[-E_{1} /(R \cdot T)\right]\right)$.

7. Physical property values normally change during reaction, due to changes in the reaction mixture composition and temperature. Calculations showed that the fluid density varies less than $2 \%$ over the entire reactor length under steady-state conditions. In this particular case, the temperature dependence of the fluid density $(\rho)$ is compensated by the composition change due to reaction. Hence, the fluid density is assumed to be constant. The Rackett equation of the ASPEN Plus program (1990) is used for the fluid density calculations.

8. The reaction enthalpy value $\left(-\Delta H_{r}\right)$ of the main reaction is used in the model. The fluid heat capacity value $\left(C_{p}\right)$ is calculated from the total temperature rise over the entire reactor length under steady-state conditions. Both properties are assumed to be constant.

9. The density $\left(\rho_{w}\right)$ and heat capacity $\left(C_{p w}\right)$ values of the construction materials of the reactor are assumed to be constant.

With these assumptions the following equations are obtained:

Component mass balances:

$$
\begin{aligned}
& \frac{\partial C_{A}}{\partial t}=-v_{t} \cdot \frac{\partial C_{A}}{\partial x}+D_{a x} \cdot \frac{\partial^{2} C_{A}}{\partial x^{2}}-k_{1} C_{B} \\
& \frac{\partial C_{B}}{\partial t}=-v_{t} \cdot \frac{\partial C_{B}}{\partial x}+D_{a x} \cdot \frac{\partial^{2} C_{B}}{\partial x^{2}}-k_{1} C_{B} \\
& \frac{\partial C_{C}}{\partial t}=-v_{t} \cdot \frac{\partial C_{C}}{\partial x}+D_{a x} \cdot \frac{\partial^{2} C_{C}}{\partial x^{2}}+k_{1} C_{B}
\end{aligned}
$$

Energy balance for the fluid:

$$
\begin{aligned}
\frac{\partial T}{\partial t}=-v_{t} \cdot \frac{\partial T}{\partial x}+\frac{\lambda_{a x}}{\rho \cdot C_{p}} \cdot \frac{\partial^{2} T}{\partial x^{2}}-\frac{4 \cdot U}{\rho \cdot C_{p} \cdot d_{1}}\left(T-T_{w}\right) & \\
& -\frac{\left(-\Delta H_{r}\right)}{\rho \cdot C_{p}} \cdot k_{1} C_{B}
\end{aligned}
$$


Energy balance for the reactor vessel:

$$
\frac{\partial T_{w}}{\partial t}=\frac{4 \cdot U \cdot d_{1}}{\rho_{w} \cdot C_{p w} \cdot\left(d_{2}^{2}-d_{1}^{2}\right)}\left(T-T_{w}\right)
$$

The fluid velocity $v_{t}$ or in dimensionless notation $\phi_{v}$ is a timedependent function, see Figure 7 . The reactor wall temperature $T_{w}$ at time $t=0$ is assumed to be equal to the fluid temperature. Hence,

Initial conditions:

$$
\begin{array}{ll}
t=0 ; & C_{A}(x, t)=C_{A}(x, 0) \\
C_{B}(x, t)=C_{B}(x, 0)=0 \\
C_{C}(x, t)=C_{C}(x, 0) \\
T(x, t)=T(x, 0) \\
T_{w}(x, t)=T_{w}(x, 0)=T(x, 0)
\end{array}
$$

The boundary conditions used by Sørensen (1976) are also used in this study to reduce the computational effort.

Boundary conditions:

$$
\begin{aligned}
t \geq 0 \text { and } x=0 ; \quad C_{A}(x, t) & =C_{A 0}(t) \\
C_{B}(x, t) & =C_{B 0}(t) \\
C_{C}(x, t) & =C_{C 0}(t)=0 \\
T(x, t) & =T_{0}(t)
\end{aligned}
$$

The parameters $v_{t}, C_{B 0}$ and $T_{0}$ can be adjusted freely within the operating limits by the plant operator or the process control system, and can be classified as manipulated or adjustable variables according to the nomenclature used in control theory (Stephanopoulos, 1984).

In a purely mathematical sense, boundary conditions are necessary to single out a particular solution to the model equations. From a physical point of view, these conditions must express the interaction between the system and its surroundings (Novy et al., 1990).

The boundary conditions used at the reactor inlet and outlet are approximations of the continuity equations at the boundaries (Fan and Ahn, 1963). The Péclet number $\left(P e=v_{t} \cdot L / D_{a x}\right)$ is estimated to be 175 , indicating that plug flow is approached (Pe > 100), according to Westerterp et al. (1984c). The Wen and Fan (1975) method is used to estimate the Péclet number:

$$
\frac{1}{B o}=\frac{3 \cdot 10^{7}}{\operatorname{Re}^{2.1}}+\frac{1.35}{\operatorname{Re}^{1 / 8}}
$$

and

$$
P e_{m}=\frac{v_{t} L}{D_{a x}}=\frac{L}{d_{1}} \cdot \frac{v_{t} d_{1}}{D_{a x}}=\frac{L}{d_{1}} \cdot B o
$$

For example, the difference between the well-known boundary conditions of Danckwerts (1953) and the boundary conditions used in this study will be negligible due to the expected high Péclet number (Fan and Ahn, 1963) and the inaccuracy of the experimental data, which will have a higher impact on the values of the estimated model parameters than the boundary conditions.

Since Eqs. 1 and 3 can be solved separately, once the solution for $C_{B}, T$ and $T_{w}$ is known it is not necessary to further consider them.

The design and operating conditions of the tubular reactor itself and the reaction system can be characterized with a certain set of dimensionless groups as shown by Westerterp and Ptasinsky (1984a), Westerterp et al. (1984b), Westerterp and Overtoom (1985), and Westerink and Westerterp (1988). According to this method, the operating and design parameters are related uniquely with the selectivity and the reaction system parameters under steady-state conditions.

Similar dimensionless groups are used in this study, and the method is extended to describe the dynamic behavior of the reactor. To do this, additional reference values are introduced for the fluid velocity $\left(v_{r}\right)$ and the reactant $B$ concentration $\left(C_{B r}\right)$, resulting in the following set of dimensionless manipulated variables:

$$
\phi_{v}=\frac{v_{i}}{v_{r}} \quad \psi_{B}=\frac{C_{B 0}}{C_{B r}} \quad \nu_{\theta}=\frac{T_{0}}{T_{r}}
$$

The values of the reference variables $v_{r}$ and $C_{B r}$ are chosen as the actual values of $v_{t}$ and $C_{B 0}$ at the steady-state condition of the reactor system at maximum plant capacity. The reference temperature $T_{r}$ is chosen as the reactor inlet temperature $T_{0}$ at the same conditions.

Different symbols are used for the dimensionless manipulated variables $\nu_{\theta}, \psi_{B}$ and $\phi_{v}$ in comparison with the symbols used for the state variables $\Gamma_{B}$ and $\theta$ to accentuate that the manipulated variables are the functions that can be adjusted freely in time. The functions of the manipulated variables $\nu_{\theta}$, $\psi_{B}$ and $\phi_{v}$ during the observed reactor startup are shown in Figures 4, 6 and 7, respectively.

Equations 2, 4 and 5 are made dimensionless by the introduction of the dimensionless quantities defined in the Notation Section. The transformed equations are:

Mass balance of component $B$ :

$$
\frac{\partial \Gamma_{B}}{\partial \sigma}=-\phi_{v} \cdot \frac{\partial \Gamma_{B}}{\partial z}+\frac{1}{P e_{m r}} \cdot \frac{\partial^{2} \Gamma_{B}}{\partial z^{2}}-D a_{r} \cdot \kappa \cdot \Gamma_{B}
$$

Energy balance for the fluid:

$$
\frac{\partial \theta}{\partial \sigma}=-\phi_{v} \cdot \frac{\partial \theta}{\partial z}+\frac{1}{P e_{h r}} \cdot \frac{\partial^{2} \theta}{\partial z^{2}}-U^{*} \cdot D a_{r}\left(\theta-\theta_{w}\right)
$$

$$
+\Delta \theta_{a d r} \cdot D a_{r} \cdot \kappa \cdot \Gamma_{B}
$$

Energy balance for the reactor vessel:

$$
\frac{\partial \theta_{w}}{\partial \sigma}=D a_{r} \cdot U^{*} \cdot \omega_{h} \cdot\left(\theta-\theta_{w}\right)
$$




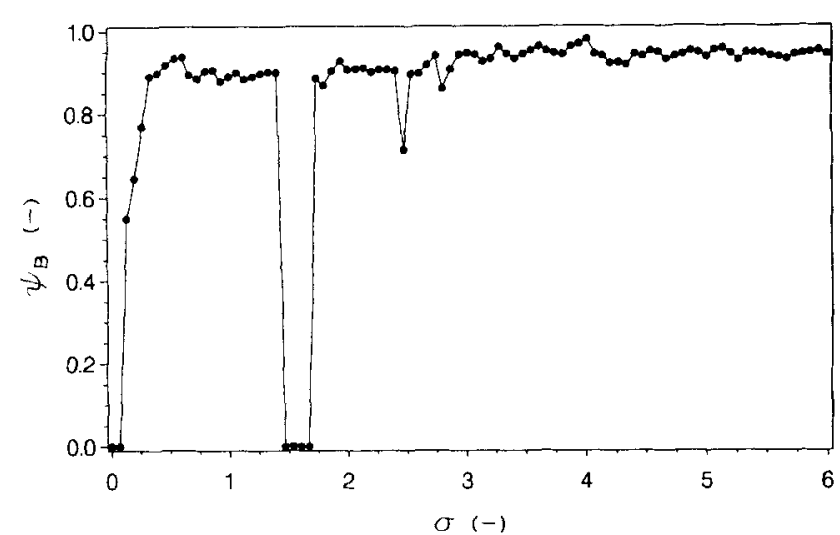

Figure 6. Calculated concentration of reactant $B$ as a function of time at the reactor inlet.

Initial conditions:

$$
\begin{aligned}
\sigma=0 ; & \Gamma_{B}(z, \sigma)=\Gamma_{B}(z, 0)=0 \\
\theta(z, \sigma) & =\theta(z, 0) \\
\theta_{w}(z, \sigma) & =\theta_{w}(z, 0)=\theta(z, 0)
\end{aligned}
$$

Boundary conditions:

$$
\begin{gathered}
\sigma \geq 0 \text { and } z=0 ; \Gamma_{B}(0, \sigma)=\Gamma_{B 0}(\sigma)=\psi_{B} \\
\theta(0, \sigma)=\theta_{0}(\sigma)=\nu_{\theta} \\
\sigma \geq 0 \text { and } z=1 ; \frac{\partial^{2} \Gamma_{B}}{\partial z^{2}}=\frac{\partial^{2} \theta}{\partial z^{2}}=0
\end{gathered}
$$

Basically Eqs. 11, 12 and 13 represent the system response as a function of the superficial fluid velocity $\phi_{v}$, the reactant $B$ inlet concentration $\psi_{B}$ and the reactor inlet temperature $\nu_{\theta}$, starting from the initial conditions at $\sigma=0$ as a reference point.

\section{Simulation and Parameter Estimation}

The method of lines is used to solve the differential equations

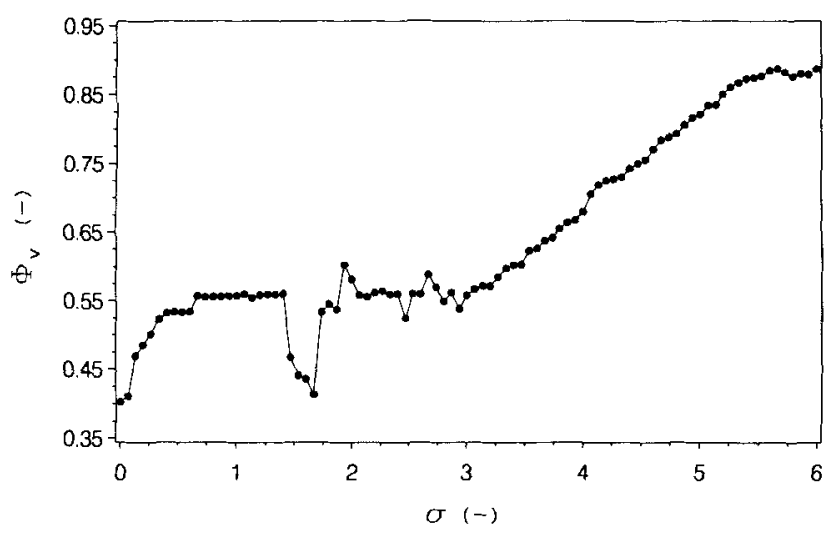

Figure 7. Calculated fluid velocity as a function of time.
(Eqs. 11, 12 and 13). Recently a review of this method is given by De Wolf (1990). This method is selected because:

1. The differential to the reactor location $z$ is eliminated by discretization and substitution of a finite difference scheme for $\partial / \partial z$. This yields a set of (continuous-time) ordinary differential equations that can be solved by standard numerical techniques.

2. The resulting set of ordinary differential equations can be solved as a function of the manipulated variables $\phi_{v}, \psi_{B}$ and $\nu_{\theta}$.

3. Ordinary differential equations solvers can easily be combined with parameter estimation techniques for system identification.

4. The method allows fast implementation in existing simulation tool boxes. The SimuSolv software package (1991) is used in this study.

A uniform grid with 501 grid points is used for discretization. The differentials of the function $f$ at grid point $i$ are replaced by the finite difference schemes:

$$
\begin{aligned}
& \frac{\partial f}{\partial z}=\frac{f(i)-f(i-1)}{\Delta z} \\
& \frac{\partial^{2} f}{\partial z^{2}}=\frac{f(i+1)-2 \cdot f(i)+f(i-1)}{\Delta z^{2}}
\end{aligned}
$$

A fifth-order, variable-step-size Runge-Kutta-Fehlberg algorithm is used to solve the resulting set of ordinary differential equations. The structure of the SimuSolv program to solve partial differential equations according to the method of lines is shown by Steiner et al. (1990a). The Akima (1970) method, which is incorporated in the SimuSolv software, is used to transform the observed discrete time values of the adjustable parameters $\phi_{v}, \psi_{B}$ and $\nu_{\theta}$ into smooth "continuous time function values." The numerical stability is provided by the correct choice of the maximum time step during integration. The Akima (1970) method is also used to generate initial values at each grid point for the resulting set ordinary differential equations by discretization of Eqs. 12 and 13, see Figure 3.

The generalized-reduced-gradient (GRG) optimization algorithm is used to estimate the parameters $\gamma, D a_{r}, P e_{m r}, P e_{h r}$, and $U^{*}$ in Eqs. 11, 12 and 13 by maximizing the Log-Likelihood objective function (see Steiner et al., 1990b, for details). The reactor temperature data set in Figure 5 is used for the parameter estimation procedure, together with the time-dependent functions of the manipulated variables $\phi_{v}, \psi_{B}$ and $\nu_{\theta}$ given in Figure 4, 6 and 7 as model input.

The GRG-algorithm is alternated by the Search algorithm to estimate new values for the parameter subset $\gamma, D a_{r}$, and $P e_{h r}$ to avoid local maxima during optimization of the LogLikelihood objective function. The parameter values resulting from the Search algorithm run are used as new starting values for an additional run with the GRG-algorithm. This parameter subset is selected from the total parameter set, because it consists of highly correlated parameters. The parameter values in Table 1 result from the final GRG-algorithm run together with the coefficient of variation $C$. V. being the standard deviation expressed as the percentage of the estimated parameter value. The overall percentage variation explained by the model is $99.57 \%$. 
Table 1. Estimated Parameter Values and C.V.-Values with $\Delta \theta_{\text {adr }}=0.34$ and $\omega_{h}=11.67$

\begin{tabular}{lcc}
\hline Parameter & Est. Value & C.V. [\%] \\
\hline$\gamma$ & 20.2 & 0.31 \\
$D a_{r}$ & 0.41 & 0.36 \\
$P e_{m r}$ & 196.0 & 0.57 \\
$P e_{h r}$ & 42.0 & 1.21 \\
$U^{*}$ & 160.0 & 1.15 \\
\hline
\end{tabular}

The calculated reactor temperature profile as a function of time over the entire reactor is presented in Figure 8, in a similar plot as the experimental temperature data, see Figure 5. The difference between the experimental and calculated temperature $\Delta \theta$ is shown in Figures 9 and 10 for different reactor locations $z$.

The calculated concentration profile of reactant $B$ as a function of time over the entire reactor is shown in Figure 11. In this figure the lines parallel to the $\sigma$-axis represent the calculated dimensionless concentration $\Gamma_{B}$ at the dimensionless location $z$, and the lines parallel to the $z$-axis connect the data at the same moment. The $\sigma$-axis is drawn from the right side to the left, and the $z$-axis is drawn in the opposite direction, which contradicts with the temperature profiles in Figures 5 and 8. Note also the breakthrough of component $B$ around time $\sigma=2$ in Figure 11.

\section{Discussion}

Process data from continuously-operated chemical plants are obtained under nonideal conditions, due to inaccuracies in the measurements of process variables and fluctuations of environmental conditions. Hence, the calculated model response within discrepancy limits between model and actual data in Figures 9 and 10 represents the dynamic behavior of this reactor system excellently.

The temperature dip caused by the reactant $B$ feed-pump failure at $\sigma \simeq 3.0$ is flattened in the system response calculated by the model, Figures 5 and 8 , for the following reasons:

- The axial dispersion coefficients are assumed to be con-

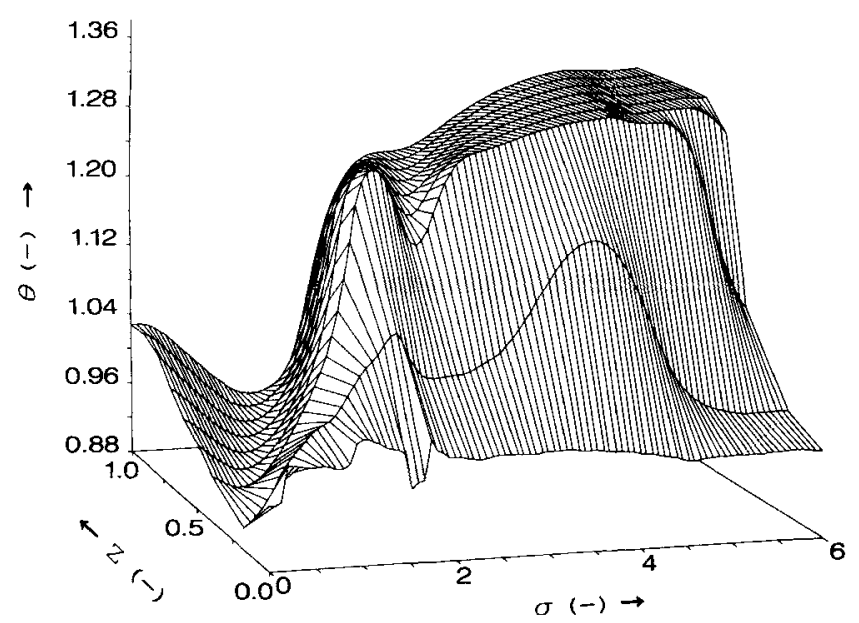

Figure 8. Calculated reactor temperature vs. reactor lo. cation and time.

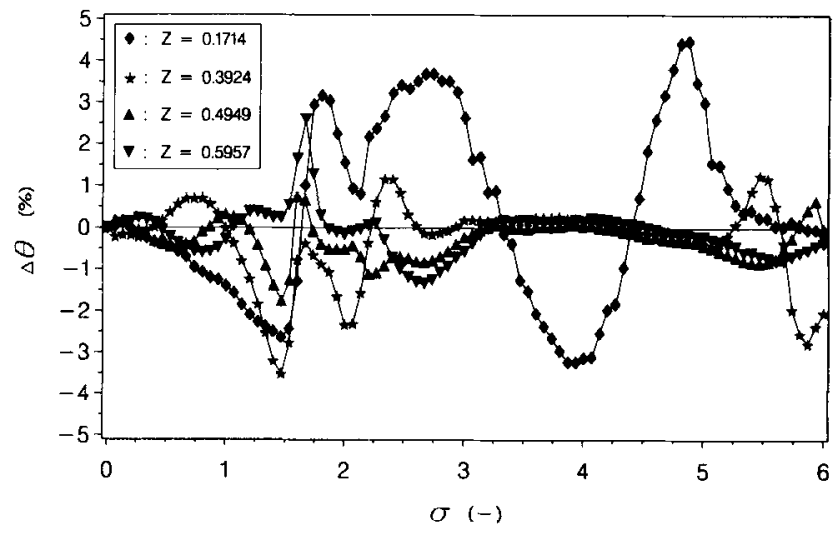

Figure 9. Experimental vs. calculated temperature as a function of time at different reactor locations.

stant over the entire reactor. This, however, is not the likely case due to the varying geometry of the system.

- The total amount of construction materials is equally distributed over the model reactor, which is not the case in the plant reactor. In the model, construction materials are oversized in the second half of the total tube length due to the difference in vessels and piping sizes.

The estimated values of $P e_{m r}$ and $P e_{h r}$ validate the approximations of the continuity equations at the system boundaries. Fan and Ahn (1963) suggest that the difference is negligible if Péclet numbers exceed a value of 30 . The difference between both Péclet numbers is great. A lower value for $\mathrm{Pe}_{h r}$ than for $P e_{m r}$ was expected, because the flow pattern induced by the baffles in the vessels create local temperature differences between both sides of the baffles, and hence a driving force for heat transport. The estimated value of $P e_{h r}$ is found to be sensitive to the geometry of the model system. Therefore, no general conclusions are drawn from the difference between the estimated values of the Péclet numbers.

In practice, the heat-transfer coefficient is high, resulting in low temperature differences between fluid and reactor wall. Hence, the assumption seems to be correct to set the initial

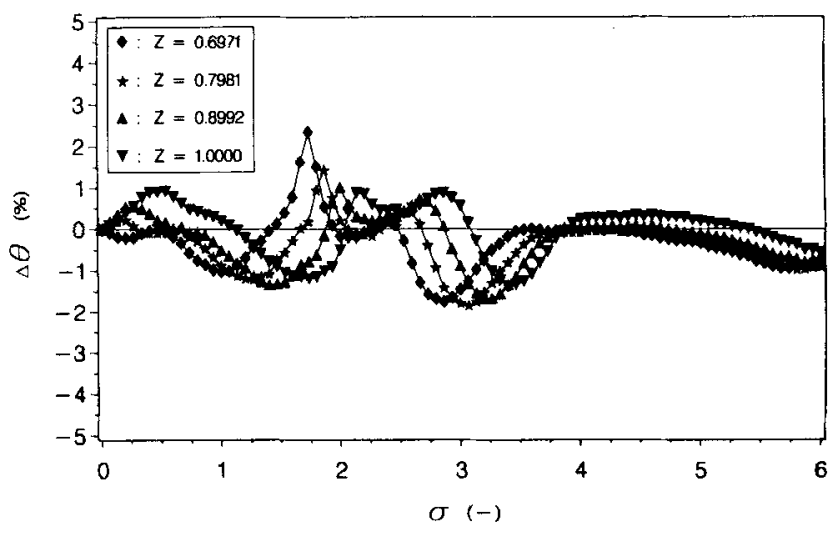

Figure 10. Experimental vs. calculated temperature as a function of time at different reactor locations. 


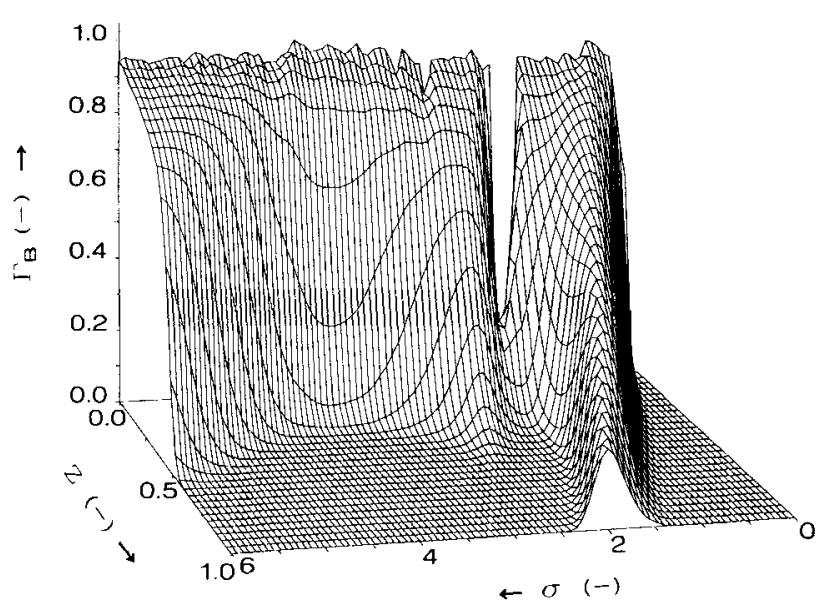

Figure 11. Calculated reactant $B$ concentration vs. reactor location and time.

values of the reactor wall temperature equal to the initial values of the fluid temperature.

Reactant $B$ should be totally converted at the reactor exit to meet the operating requirements of this reactor system, which is not the case during a certain period at $\sigma \simeq 2$. This reactant breakthrough can have a significant impact on the operation of the downstream plant section of this reactor system and depends on the total amount of reactant $B$ fed to this section. Its detailed discussion is beyond the scope of this article.

If the restart of the reactant $B$ feed-pump after the pump failure is considered as a second startup, a significant difference can be observed in the reactant $B$ concentration profiles in Figure 11. This difference stems from the following two reasons:

- The average reactor temperature during the second startup is much higher than for the first one.

- The reactant $B$ feed is not ramped to its setpoint during the second startup, see Figure 2.

The combination of these effects results in a higher average reaction rate due to a higher initial temperature level and a higher initial concentration of reactant $B$ for the second startup, resulting in a complete conversion of reactant $B$ at $z \simeq$ 0.60 .

Equations 11, 12 and 13 represent the system response as a function of the manipulated variables, starting from the initial conditions as a reference point:

- Here, initial is not necessarily defined as the state of the system at $\sigma=0$, but can also be defined as the state of the system at time $\sigma$ when the reactant $B$ feed-pump is put into service.

- In the presented example, there are two startups with different initial temperature profiles due to the feed-pump failure. In the first case, nonconverted reactant $B$ ends up in the reactor effluent, which is not the case during the second startup.

It can be conciuded that the initial temperature profile over the entire reactor should meet a certain criterion to ensure a complete conversion of reactant $B$ during reactor startup. The initial reactor temperature should be higher than the normal reactor inlet temperature. This is in contrast with the first idea to start up the feed pump of the second reactant when the normal reactor inlet temperature conditions are obtained. A "similar effect" is known from limit cycle behavior of cooled, continuously-operated stirred-tank reactors. As soon as a temperature limit cycle starts in this type of reactors, the reactor temperature has to be increased to suppress the temperature fluctuations (Heemskerk et al., 1980). This is in contrast to the first impulse of the operator who normally would increase the cooling medium flow rate to suppress temperature fluctuations, but with an opposite result! The risk of excessive high temperatures and temperature runaway behavior of reactor systems is well known. But a decrease in reactor temperature does not always result in an increase in process safety! For the reactor system studied here, much higher initial temperature levels are required than was expected to ensure a complete reactant $B$ conversion during startup.

To determine the required initial reactor temperature profile regarding the reactant $B$ conversion criterion, several aspects should be taken into account:

- The influence of the heat take-up in the reactor vessel wall, which is determined by the variables $\omega_{h}$ and $U^{*}$

- The influence of the mass and heat dispersion numbers $P e_{m r}$ and $P e_{h r}$, and the value of the Damköhler number $D a_{r}$.

The trajectories of the manipulated variables $\phi_{v}, \psi_{B}$ and $\nu_{\theta}$, which describe these variables as a function of time, should keep the system within the operating limits. Of course, the required trajectories can be defined only in relation to the initial conditions of the reactor itself and with the operating requirements taken into account. A reactor startup cannot be isolated from a plant startup. Therefore, the operating procedures for a reactor startup should be defined within the boundaries of a total plant startup, taking into account: 1 . how the initial temperature profile can be achieved in the reactor without the heat of reaction available; 2 . The downstream plant section of the reactor system should be able to process the reactor effluent, also during startup, when the reactor effluent conditions are not necessarily the same as the process conditions during normal operation.

\section{Conclusions}

A dynamic model of an industrial reactor system is presented and the system response is calculated as a function of experimentally-determined, time-dependent manipulated variables, which includes a failure of the feed pump of one of the reactants during startup. The values of the model parameters are estimated by using the SimuSolv (1991) computer program. The data set collected during the reactor startup is used for the parameter estimation procedure. An excellent agreement is obtained between the experimental and the calculated system response.

Many continuously-operated industrial reactor systems require a complete conversion of one of the main reactants at the reactor exit. It is shown that before this main reactant is fed into the reactor, the temperature profile over the entire reactor should meet a certain criterion. For the presented example, the initial reactor temperature should be much higher than was expected, compared to the reactor inlet temperature during normal steady-state operation. This is to ensure a com- 
plete conversion of the main reactant during reactor startup, which is one of the requirements for process safety. Whether reactor temperatures during startup must be higher as a generally valid rule will have to be the subject of much further research.

Declarative knowledge and procedural knowledge can be distinguished in plant-wide operations (Stephanopoulos, 1988). Both types of knowledge are required to study the operational aspects of a reactor startup. A dynamic process model, based on first principles, should answer questions about reactor behavior and operating limits. A representation of the operating procedures should answer questions about "how" and "when" to start up a reactor system, because a reactor startup cannot be isolated from an entire plant startup. Some research results on the synthesis of reactor operating procedures for the startup of continuously-operated chemical plants will be discussed in a future article.

\section{Notation}

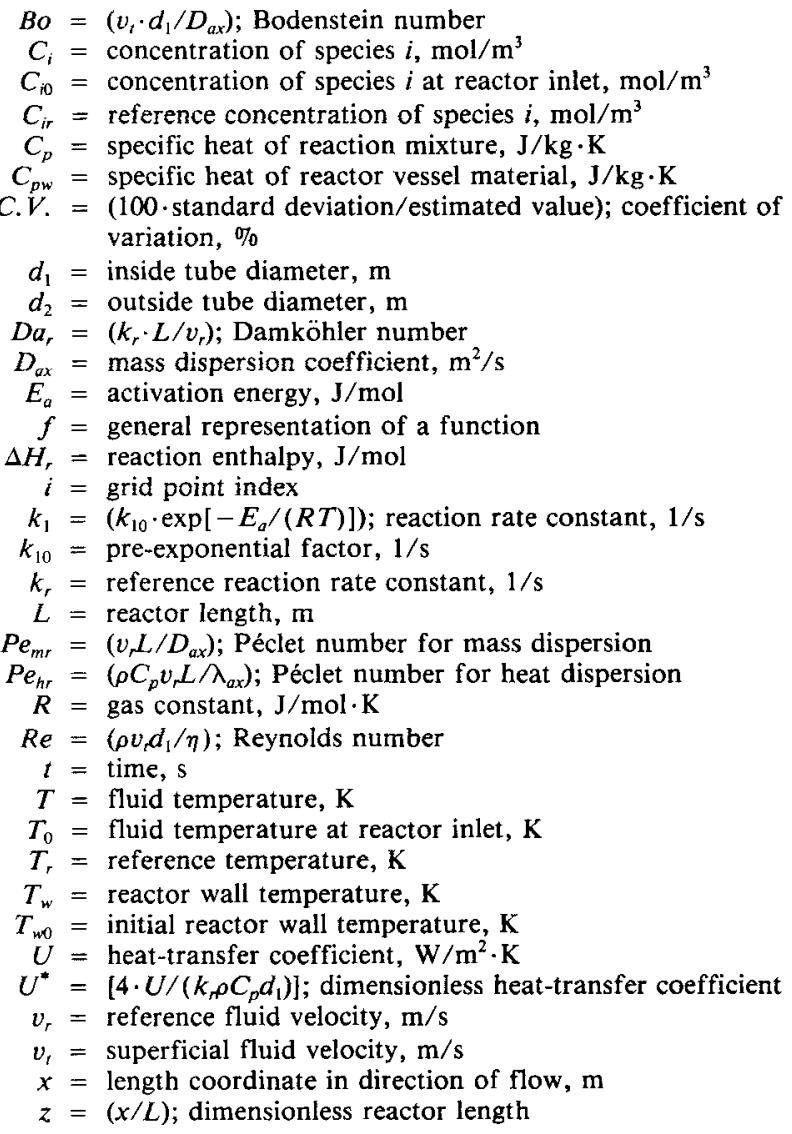

\section{Greek letters}

$$
\begin{aligned}
\gamma= & {\left[E_{a} /\left(R \cdot T_{r}\right)\right] ; \text { dimensionless activation temperature } } \\
\Gamma_{B}= & \left(C_{B} / C_{B r}\right) ; \text { dimensionless concentration reactant } B \\
\Gamma_{B 0}= & \left(C_{B 0} / C_{B r}\right) ; \text { dimensionless concentration reactant } B \text { at the } \\
& \text { reactor inlet } \\
\eta= & \text { fluid viscosity, } \mathrm{kg} / \mathrm{m} \cdot \mathrm{s} \\
\theta= & \left(T / T_{r}\right) ; \text { dimensionless fluid temperature } \\
\theta_{w}= & \left(T_{w} / T_{r}\right) ; \text { dimensionless reactor wall temperature } \\
\theta_{0}= & \left(T_{0} / T_{r}\right) ; \text { dimensionless reactor inlet temperature } \\
\Delta \theta_{a d r}= & {\left[\left(-\Delta H_{r}\right) C_{B r} /\left(\rho C_{p} T_{r}\right)\right] ; \text { dimensionless adiabatic tempera- } } \\
& \text { ture rise }
\end{aligned}
$$

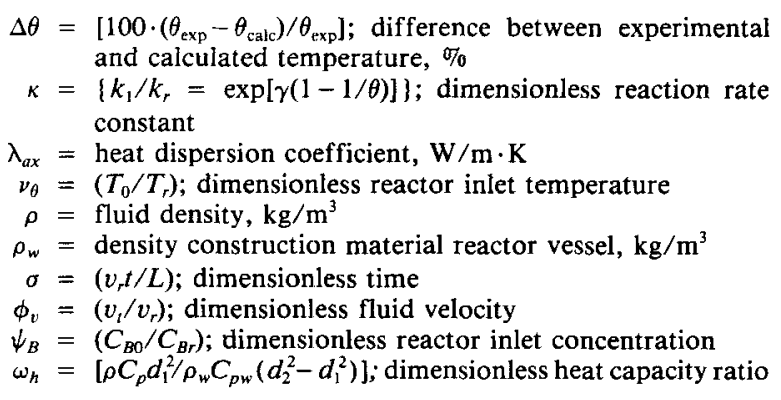

\section{Subscripts}

$$
\begin{aligned}
A & =\text { reactant } A \\
B & =\text { reactant } B \\
C & =\text { product } C \\
\text { calc } & =\text { calculated value } \\
\text { exp } & =\text { experimental value }
\end{aligned}
$$

\section{Literature Cited}

Akima, H., "A New Method of Interpolation and Smooth Curve Fitting Based on Local Procedures," J. of the Assoc. for Computing Machinery, 17, 589 (1970).

Amundson, N. R., et al., Frontiers in Chemical Engineering: Research Needs and Opportunities, National Academy Press, Washington, DC (1988).

ASPEN Plus Flowsheet Simulation Program, Aspen Technology Inc., Cambridge, MA, Version 8.3 (1990).

Danckwerts, P. V., "Continuous Flow Systems: Distribution of Residence Times," Chem. Eng. Sci, 2, 1 (1953).

Fan, L. T., and Y. K., Ahn, "Frequency Response of Tubular Flow Systems," Chem. Eng. Prog. Symp. Ser., 46, 91 (1963).

Haastrup, P., "Design Error in the Chemical Industry,"' I. Chem. Eng. Symp. Ser., Loss Prevention and Safety Promotion, No. 80 , Vol. 1, J15 (1983).

Hahn, D. R., L. T. Fan, and C. L. Hwang, “Optimal Start-Up Control of a Jacketed Tubular Reactor," AIChE J., 17, 1394 (1971).

Heemskerk, A. H., W. R. Dammers, and J. M. H. Fortuin, "Limit Cycles Measured in a Liquid-Phase Reaction System," Chem. Eng. Sci., 35, 439 (1980).

Mann, R., I. J. Gardner, and C. Morris, "Sulphur Dioxide Emissions During Start-Up of a Contact Sulphuric Acid Plant,"' I. Chem. E., Symp. Ser., 57, L1 (1979).

Mann, R., I. J. Gardner, and C. Morris, "Reactor Dynamics and Strategies for Minimising $\mathrm{SO}_{2}$ Emissions During Start-Up of a Contact Sulphuric Acid Plant,"' Chem. Eng. Sci., 35, 185 (1980).

Mann, R., E. Stavridis, and K. Djamarani, "Experimental Fixed-Bed Reactor Dynamics for $\mathrm{SO}_{2}$ Oxidation," Chem. Eng. Res. Dev., 64, 248 (1986).

Novy, R. A., H. T. Davis, and L. E. Scriven, "Upstream and Downstream Boundary Conditions for Continuous-Flow Systems," Chem. Eng. Sci., 45, 1515 (1990).

SimuSolv-Modeling and Simulation Software, The Dow Chemical Company, Midland, MI, Version 2.1-55 (1991).

Sørensen, J. P., "Experimental Investigation of the Dynamics of a Fixed-Bed Reactor," Chem. Eng. Sci., 31, 719 (1976).

Steiner, E. C., T. D. Rey, and P. S. McCroskey, SimuSolv Reference Guide," The Dow Chemical Company, Midland, MI, Vol. 1 (1990a).

Steiner, E. C., T. D. Rey, and P. S. McCroskey, SimuSolv Reference Guide, The Dow Chemical Company, Midland, MI, Vol. 2 (1990b).

Stephanopoulos, G., Chemical Process Control: an Introduction to Theory and Practice, Prentice Hall, London (1984).

Stephanopoulos, G., "The Scope of Artificial Intelligence in PlantWide Operations," Foundations of Computer-Aided Process Operations, G. V. Reklaitis and D. Spriggs, eds., CACHE-Elsevier, Austin, (1988).

Wen, C. Y., and L. T. Fan, Models for Flow Systems and Chemical Reactors, Marcel Dekker, New York (1975). 
Westerink, E. J., and K. R. Westerterp, "Safe Design of Cooled Tubular Reactors for Exothermic, Multiple Reactions: Multiple Reaction Networks," Chem. Eng. Sci., 43, 1051 (1988).

Westerterp, K. R., and K. J. Ptasinski, "Safe Design of Cooled Tubular Reactors for Exothermic, Multiple Reactions, Parallel Reactions, and Development of Criteria," Chem. Eng. Sci., 39, 235 (1984a).

Westerterp, K. R., K. J. Ptasinsky, and R. R. M. Overtoom, "Safe Design of Cooled Tubular Reactors for Exothermic, Multiple First Order Reactions," ACS Symp. Ser., No. 237, pp. 323-335 (1984b). Westerterp, K. R., W. P. M. van Swaaij, and A. A. C. M. Beenackers,
Chemical Reactor Design and Operation, Wiley, Chichester U.K., 2nd ed. (1984c).

Westerterp, K. R., and R. R. M., Overtoom, "Safe Design of Cooled Tubular Reactors for Exothermic, Multiple Reactions: Consecutive Reactions," Chem. Eng. Sci., 40, 155 (1985).

Wolf, S. de, "Modelling, System Identification and Control of an Evaporative Continuous Crystallizer," PhD Thesis, Delft Univ. of Technology (1990).

Manuscript received Jan. 23, 1992, and revision received June 18, 1992. 